\title{
Robust distributed control of quasilinear reaction-diffusion equations via infinite-dimensional sliding modes *
}

\author{
Andrea Cristofaro ${ }^{\text {a,b }}$ \\ ${ }^{a}$ Department of Technology Systems, Faculty of Mathematics and Natural Sciences, University of Oslo, 2007 Kjeller, Norway \\ ${ }^{\mathrm{b}}$ School of Science and Technology - Mathematics Division, University of Camerino, 62032 Camerino, Italy
}

\begin{abstract}
This paper investigates the problem of robust tracking control for quasilinear reaction-diffusion partial differential equations subject to external unknown perturbations. The considered class of equations is quite general, and includes classical equations such as the heat equation or the Fisher-KPP equation as special cases. Global practical stabilization of the tracking error system is established under mild conditions on the disturbance term using a regularized infinite-dimensional sliding-mode controller. Extensive simulations support and validate the theoretical results.
\end{abstract}

Key words: Distributed-parameter systems, reaction-diffusion equations, sliding-mode control

\section{Introduction}

Reaction-diffusion equations are second-order parabolic differential equations which are well suited to describe chemical reactions, pattern formation and population dynamics [14], [16]. In recent years, partial differential equations have largely attracted the attention of the control community (see for instance [13], [19], [20] and the references therein) since many plant models are described by infinite-dimensional systems, hence involving PDEs or systems of PDEs: the fields of application encompass robotics (haptic controllers and flexible manipulators), industrial processes (manufacturing, reactors and heat transfer plants) as well as the biomedical domain (tissue engineering).

Due to the high complexity of such models, several sources of uncertainty are likely to be encountered, this bringing the focus on the analysis and synthesis of robust control strategies. Sliding-mode [32] is a well established robust control technique having the advantage of constraining the state of the controlled system in a region which results to be invariant with respect to external disturbances. Sliding-mode controllers have also been proposed to tackle the problem of robust control for PDEs [31], [25], [29]. In particular in [31]

\footnotetext{
^ A preliminary version of this paper was presented at IFAC World Congress 2014, Cape Town, ZA.

Email address: andrea.cristofaro@its.uio.it (Andrea Cristofaro).
}

the problem of distributed control for quasilinear firstorder parabolic equations is addressed and a variablestructure control law is proposed, while in [26], [29] the authors focus on the design of sliding-mode controllers for robust tracking in the case of unidimensional heat equation and wave equation.

In the framework of reaction-diffusion equations, both boundary control [3] and distributed control [17] have been investigated. This paper focuses on the robust asymptotic tracking problem for reaction-diffusion equations in multidimensional space domains subject to external bounded disturbances, i.e. the control objective is to reject the disturbance and steer the solution towards a desired reference profile. We consider quasilinear equations in divergence form, i.e. equations which are linear in second-order derivatives, with a possible nonlinear reaction term. Both the classical heat equation and the well known Fisher-KPP equation are included in such class of problems as special cases.

Given a reference function to be tracked, robust global practical stability of the tracking error system is proved via a regularized sliding-mode controller. The main advantage of the proposed approach is that, thanks to the regularization of the sliding-mode, no singularity arises in the control variable, this allowing to avoid the introduction of approximate solutions. While the regularized sliding-mode control leads in general to practical stabilization only, it must be noticed that, by suitably tuning the controller parameters, the reference profile can be robustly tracked with any desired accuracy. 
The paper is structured as follows. In Section 2 the model setup is presented, while Sections 3 and 4 contain the main results: design of the robust tracking control law in the presence of bounded external perturbations and regularization of the sliding-mode controller with practical stability guarantees. Extensive numerical simulations supporting the theoretical developments are reported in Section 5.

\section{Model and setup}

Let $\Omega \subset \mathbb{R}^{n}, n \geq 1$, be a bounded open set and consider the infinite-dimensional differential problem

$$
\begin{gathered}
\partial_{t} h=L(t, x, h)+g(h)+u(t, x)+b(t, x) \\
t \in(0,+\infty), \quad x \in \Omega
\end{gathered}
$$

with Dirichlet boundary conditions

$$
h(t, \cdot)_{\left.\right|_{\partial \Omega}}=h_{\diamond}(t)
$$

and initial condition

$$
h(0, x)=h_{\bullet}(x)
$$

The domain $Q:=(0,+\infty) \times \Omega$ is referred to as parabolic cylinder. The linear operator $L(t, x, \cdot)$, which models the diffusion term, is assumed to be uniformly elliptic [10], and its form will be specified further on. The lower-order term $g(u)$ is the reaction term in the equation, and it is allowed to enclose nonlinearities. The function $u(t, x)$ represents a distributed control input, while $b(t, x)$ is an unknown disturbance, such as an external uncertain source.

Assumption 1 The operator $L(t, x, \cdot)$ is in divergence ${ }^{1}$ form

$$
L(t, x, h)=\operatorname{div}(A(t, x) \nabla h),
$$

where $A(t, x)$ is a symmetric matrix function such that there exist positive constants $\lambda_{\min }, \lambda_{\max } \in \mathbb{R}^{+}$with

$$
\lambda_{\min }|\xi|^{2} \leq\langle\xi, A(t, x) \xi\rangle \leq \lambda_{\max }|\xi|^{2}
$$

for any $(t, x) \in Q$ and any $\xi \in \mathbb{R}^{n}$.

Assumption 2 The reaction term satisfies the following growth condition: there exist two continuous functions $\beta, \sigma: \mathbb{R} \rightarrow \mathbb{R}^{+} \cup\{0\}$ with $\beta(0)=0$ such that

$$
(\zeta-z)(g(\zeta)-g(z)) \leq(\beta(\zeta-z)+\sigma(z))|\zeta-z|^{2}
$$

for any $\zeta, z \in \mathbb{R}$.

1 We recall that, for a given vector field $F(x)=$ $\left[F_{1}(x), \cdots, F_{n}(x)\right]$, the divergence operator is defined by $\operatorname{div} F(x)=\partial_{x_{1}} F_{1}(x)+\partial_{x_{2}} F_{2}(x)+\cdots+\partial_{x_{n}} F_{n}(x)$.
Let us notice that, in particular, any Lipschitz function $g(\cdot)$ satisfies Assumption 2. The proposed model takes inspiration from a classical equation arising in population dynamics and also used in nuclear reactions modeling, namely the Fisher-Kolmogorov-Petrovsky-Piskunov or Fisher-KPP equation [14]

$$
\partial_{t} h=\Delta h+h(1-h),
$$

which is a special case of (1) with $A(t, x) \equiv I$ and $g(\zeta)=\zeta(1-\zeta)$. Let us stress that the growth conditions proposed in Assumption 2 are indeed tailored on the reaction term of such model equation.

For the readers' convenience, we recall the following classical definitions of functional spaces with $p \geq 1$ :

$$
\begin{gathered}
\mathcal{L}^{p}(\Omega):=\left\{f(\cdot):\|f\|_{p}:=\left(\int_{\Omega}|f(x)|^{p} d x\right)^{1 / p}<\infty\right\}, \\
\mathcal{L}^{\infty}(\Omega):\left\{f(\cdot):\|f\|_{\infty}:=\operatorname{ess} \sup _{\Omega}|f(x)|<\infty\right\}, \\
W^{k, p}(\Omega):=\left\{f(\cdot):\left\|f^{(j)}\right\|_{p}<\infty \forall j=0,1, \ldots, k\right\},
\end{gathered}
$$

where $f^{(j)}(x)$ denotes the (weak) $j^{\text {th }}$-derivative of the function $f(x)$. Throughout the paper we will work with strong solutions $h(t, x)$ to (1) with the following integral regularity

$$
\begin{aligned}
& h(t, \cdot) \in W^{2,2}(\Omega) \forall t \in[0, \infty), \\
& h(\cdot, x) \in W^{1,2}(0, \infty) \forall x \in \Omega .
\end{aligned}
$$

Assumption 3 A positive number $M_{b}$ can be (a priori) determined such that

$$
\|b(t, \cdot)\|_{2} \leq M_{b} \quad \forall t \geq 0
$$

Moreover, there exists a closed linear subspace $\mathcal{W} \subset$ $\mathcal{L}^{2}(\Omega)$ such that

$$
b(t, \cdot) \in \mathcal{W} \quad \forall t \geq 0 .
$$

Let us denote by $P_{\mathcal{W}}$ and $P_{\mathcal{W}}^{\perp}$ the linear projection operators associated to the subspace $\mathcal{W}$; in particular given an arbitrary function $\varpi(\cdot) \in \mathcal{L}^{2}(\Omega)$, it admits a unique decomposition [5]

$$
\varpi(\cdot)=P_{\mathcal{W}} \varpi(\cdot)+P_{\mathcal{W}}^{\perp} \varpi(\cdot),
$$

with $P_{\mathcal{W}} \varpi(\cdot) \in \mathcal{W}$ and $\int_{\Omega} P_{\mathcal{W}}^{\perp} \varpi(x) p(x) d x=0$ for any function $p(\cdot) \in \mathcal{W}$.

Let us consider a reference function

$$
h^{\star}(t, x): Q \rightarrow \mathbb{R}
$$


with integral regularity

$$
\begin{aligned}
& h^{\star}(t, \cdot) \in W^{2,2}(\Omega) \forall t \in[0, \infty), \\
& h^{\star}(\cdot, x) \in W^{1,2}(0, \infty) \forall x \in \Omega
\end{aligned}
$$

and such that the boundary conditions are consistent with those of the original problem Cauchy-Dirichlet differential problem, i.e.

$$
h^{\star}(t, \cdot)_{\left.\right|_{\partial \Omega}}=h_{\diamond}(t) .
$$

Problem 1 - Robust asymptotic tracking. The basic task of the considered control problem is to design the input $u(t, x)$ in order to track the reference $h^{\star}(t, x)$, i.e. such that the following asymptotic condition is verified

$$
\begin{aligned}
& \lim _{t \rightarrow \infty} \int_{\Omega}\left|h(t, x)-h^{\star}(t, x)\right|^{2} d x \\
= & \lim _{t \rightarrow \infty}\left\|h(t, \cdot)-h^{\star}(t, \cdot)\right\|_{2}^{2}=0
\end{aligned}
$$

For notational convenience, let us introduce the tracking error $q(t, x)=h(t, x)-h^{\star}(t, x)$. The error dynamics is then assigned by

$$
\begin{aligned}
\partial_{t} q & =L(t, x, q)+g(h)-g\left(h^{\star}\right)-\partial_{t} h^{\star} \\
& +R\left(t, x, h^{\star}\right)+u(t, x)+b(t, x),
\end{aligned}
$$

where the "reminder" operator $R(t, x, \cdot)$ is defined as $R(t, x, v)=L(t, x, v)+g(v)$ for any $v \in W^{2,2}(\Omega)$.

We point out that, by construction, the function $q(t, x)$ verifies Dirichlet boundary conditions $q(t, \cdot)_{\left.\right|_{\partial \Omega}}=0$. Prior to introduce the main sections of the paper, we recall the following classical result for parabolic partial differential equations (see [10], Thm 2.3.2 and Thm 2.3.5). Let us also mention that an existence and uniqueness theory for reaction-diffusion equations can be found in [30].

Theorem 1 Let us fix arbitrarily $T>0$ and consider the bounded parabolic cylinder $Q_{T}:=(0, T) \times \Omega$. Assume that the function $p(t, x): \bar{Q}_{T} \rightarrow \mathbb{R}$ verifies

$$
\begin{aligned}
& p(t, x) \in C\left(\bar{\Omega}_{T}\right), \\
& p(t, \cdot) \in C^{2}(\Omega) \forall t \in[0, T], \\
& p(\cdot, x) \in C^{1}(0, T) \forall x \in \Omega .
\end{aligned}
$$

Then, for any $z_{0}(x) \in C^{2}(\Omega)$, there exists a unique strong solution $z(t, x) \in C^{2}\left(Q_{T}\right)$ of the linear parabolic problem

$$
\left\{\begin{array}{l}
\partial_{t} z=L(t, x, z)+p(t, x) \quad(t, x) \in Q_{T} \\
z(t, \cdot)_{\left.\right|_{\partial \Omega}}=0 \quad \forall t \in[0, T] \\
z(0, x)=z_{0}(x) \quad \forall x \in \Omega .
\end{array}\right.
$$

\section{Tracking control design}

As for the case of finite-dimensional linear systems, output regulation [6] is a standard and powerful approach to output reference tracking with disturbance decoupling. In particular, for parabolic equations with boundary control, output regulation has been proposed using backstepping [22] [9] and dual observers [8]. However, the linearity of the equations and the internal model principle [28], i.e. the necessity of dealing with references generated by a linear exosystem, are inherent limitations of the output regulation approach. Alternatively, adaptive disturbance rejection has been proposed for several classes of linear or quasilinear evolution equations with boundary control [18] [15] [4] [2] both in the collocated and the anti-collocated setup. The basic idea of adaptive cancellation is to estimate the disturbance first, and then to add a suitable correction signal in the control input. In our case, the nonlinear parabolic equation (1) features distributed control and disturbance, and Problem 1 consists in the full-state tracking of an arbitrary reference, i.e. no exosystem is considered. In order to achieve this goal, we focus on the robust stabilization of error system (3). In finite-dimensional systems slidingmodes have been largely recognized as a powerful tool for achieving robust stabilization in the presence of matched disturbances, and this motivates the idea of adopting a similar approach to tackle Problem 1. It is worth to anticipate that using sliding-mode control in infinitedimensional systems is far from being trivial and may lead to ill-posed equations in principle; such issue and the design of an enhanced control law to overcome it are extensively discussed in Remark 5 and Section 4.

Let us first discuss the stabilization of the error system by Lyapunov methods and the introduction of novel control laws based on advanced power integrability analysis.

As the control objective is to steer the error function $q(t, x)$ to the origin, and such function belongs to the space $\mathcal{L}^{2}(\Omega)$ at any given time instant, it is natural to consider the Lyapunov function candidate (see [24])

$$
V(q(t, \cdot))=\frac{1}{2} \int_{\Omega}|q(t, \cdot)|^{2} d x=\frac{1}{2}\|q(t, \cdot)\|_{2}^{2}
$$

Deriving $V$ along the system trajectory yields (from now the dependency on $(t, x)$ will be dropped when possible)

$$
\begin{aligned}
\dot{V}(q(t, \cdot)) & =\int_{\Omega}\left[\partial_{t} q \cdot q\right] d x \\
& =\int_{\Omega} L(t, \cdot, q) q d x+\int_{\Omega}\left(g(h)-g\left(h^{\star}\right)\right) q d x \\
& +\int_{\Omega}\left(-\partial_{t} h^{\star}+R\left(t, \cdot, h^{\star}\right)\right) q d x+\int_{\omega} u q d x \\
& +\int_{\Omega} b q d x
\end{aligned}
$$


By the divergence lemma, recalling that $q(t, x)$ satisfies equation (3) with homogeneous Dirichlet conditions and denoting by $\mathbf{n}$ the outer normal vector to $\partial \Omega$, one gets

$$
\begin{aligned}
\int_{\Omega} L(t, \cdot, q) q d x & =\underbrace{\int_{\partial \Omega}\langle A(t, \cdot) \nabla q, \mathbf{n}\rangle q d x}_{=0} \\
& -\int_{\Omega}\langle A(t, \cdot) \nabla q, \nabla q\rangle d x \\
& \leq-\lambda_{\min } \int_{\Omega}|\nabla q|^{2} d x \\
& \leq-\lambda_{\min } C_{\mathrm{P}} \int_{\Omega}|q|^{2} d x
\end{aligned}
$$

where the last two inequalities follow from Assumption and the Poincarè inequality, respectively. Moreover, focusing on the second term in the right-hand side of (4) one has

$$
\int_{\Omega}\left(g(h)-g\left(h^{\star}\right)\right) q d x \leq \int_{\Omega}\left(\beta(q)+\sigma\left(h^{\star}\right)\right) q^{2} d x
$$

Such derivations suggest that a good choice for the nominal control input might be the following:

$$
u_{\circ}=-\left(\eta+\beta(q)+\sigma\left(h^{\star}\right)\right) q+\partial_{t} h^{\star}-R\left(t, \cdot, h^{\star}\right)
$$

with $\eta \geq 0$. Let us stress that the latter control input is the superposition of an error feedback with a feedforward control, i.e. $u_{\circ}$ only depends on the tracking error $q$ and the reference function $h^{\star}$ together with its derivatives. The existence of a unique solution for the error equation (3) driven by (7) is granted by Theorem 1 up to small adjustments. Furthermore, the following stability result is straightforward to prove.

Proposition 1 Assume that no disturbances enter the system, i.e. $b(t, x) \equiv 0$. Then the origin $\{q=0\}$ is exponentially stable for the system driven by $u_{\circ}(t, x)$, with a convergence rate $\kappa \geq 2\left(\eta+\lambda_{\min } C_{\mathrm{P}}\right)$.

PROOF. Consider the Lyapunov function candidate $V(q(t))$ and compute its derivative as in (4). Using the majorizations obtained in (5) and (6), and applying the control input (7) one gets

$$
\begin{aligned}
\dot{V}(q(t, \cdot)) & \leq-\left(\eta+\lambda_{\min } C_{\mathrm{P}}\right) \int_{\Omega}|q(t, \cdot)|^{2} d x \\
& =-2\left(\eta+\lambda_{\min } C_{\mathrm{P}}\right) V(q(t, \cdot))
\end{aligned}
$$

which guarantees exponential stability by a standard Lyapunov argument. In particular, the tracking performance

$$
\left\|h(t, \cdot)-h^{\star}(t, \cdot)\right\|_{2}^{2} \leq e^{-2\left(\eta+\lambda_{\min } C_{\mathrm{P}}\right) t}\left\|h_{\bullet}(\cdot)-h^{\star}(0, \cdot)\right\|_{2}^{2}
$$

is enforced.

\subsection{Advanced control features}

It is interesting to note that, addressing a finer analysis of the power integrability of solutions, the stabilizing control law (7) can be enhanced in a few useful ways.

Remark 2 In the presence of input limitations, a more clever choice can be made by letting $\eta$ be state-dependent and allowing it to assume negative values. For instance, exponential stability is still preserved by taking $\eta=\eta(q)$ such that

$$
\sup _{q \in \mathbb{R}}\left\{\lambda_{\min } C_{\mathrm{P}}+\eta(q)-\beta(q)\right\} \geq \varrho
$$

where $\varrho>0$ is an arbitrarily small constant.

Proposition 3 Assume that a power function bound $\beta(q) \leq c_{1}|q|^{\alpha}$ with $c_{1}, \alpha>0$ holds, and that the Sobolev conjugate $^{2}$ statisfies

$$
2^{*}=\frac{2 N}{N-2} \geq 2+\alpha .
$$

with $N>2$. Then, if the ellipticity bound $\lambda_{\min }$ for the differential operator $L(t, x, q)$ is sufficiently large, the reduced control input

$$
u_{\circ}=-\left(\eta+\sigma\left(h^{\star}\right)\right) q+\partial_{t} h^{\star}-R\left(t, \cdot, h^{\star}\right)
$$

guarantees asymptotic stabilization.

PROOF. Under the given assumptions, some finer estimates can be established based on the classical Gagliardo-Nirenberg-Sobolev inequality (see Appendix A), this allowing a considerable lightening of the control action. In fact, using the interpolation inequality $^{3}|q|^{\alpha} \leq \frac{1+|q|^{2^{*}-2}}{\gamma\left(\alpha, 2^{*}-2\right)}$, rearranging and isolating the significant terms one has

$$
\begin{aligned}
& \int_{\Omega} L(t, \cdot, q) q d x+\int_{\Omega} \beta(q) q^{2} d x \\
\leq & -\lambda_{\min } \int_{\Omega}|\nabla q|^{2} d x+c_{1} \int_{\Omega}|q|^{2+\alpha} \\
\leq & -\left(\lambda_{\min } C_{\mathrm{S}} \theta-\frac{c_{1}}{\gamma\left(\alpha, 2^{*}-2\right)}\right) \int_{\Omega}|q|^{2^{*}} d x \\
& -\left(\lambda_{\min } C_{\mathrm{P}}(1-\theta)-\frac{c_{1}}{\gamma\left(\alpha, 2^{*}-2\right)}\right) \int_{\Omega}|q|^{2} d x
\end{aligned}
$$

for any $\theta \in(0,1)$. Now, if $\lambda_{\min }$ is large enough, a suitable $\theta \in(0,1)$ can be found as to make the quantities in brackets simultaneously positive and enforce the Lyapunov stability condition.

\footnotetext{
${ }^{2}$ Given $1<p<N$, its Sobolev conjugate $p^{*}$ is defined as the number such that $1 / p^{*}=1 / p-1 / N$.

3 See Appendix B
} 
The above result shows in particular that the diffusion term $L(t, x, q)$ of the equation may be capable to compensate for the "bad" term $\beta(q) q$ without the need of a dedicated control action.

Remark 4 When, conversely, no input bounds are imposed, there is margin to enhance the control performances, e.g. finite-time stabilization may be enforced. This can be achieved by introducing an additional term in the control input, having a power fractional structure. A similar procedure, which can be inherited here, is described in [29] and [7].

\subsection{Sliding-mode control}

The underlying idea of classical, finite dimensional sliding-modes is to constrain the evolution of the system, by means of discontinuous control actions, onto a manifold to be suitably shaped in order to result somewhat orthogonal to the directions of disturbances. Consequently, as long as such discontinuous control action is enforced and the disturbances remain bounded, the motion along the sliding manifold results to be invariant to the disturbances. Let us attempt mimicking the same approach in an infinite-dimensional setting by the use of unit inputs [27]. To this end, let us consider the discontinuous control input

$$
v_{b}(t, x)=-M_{b} \frac{P_{\mathcal{W}} q(t, x)}{\left\|P_{\mathcal{W}} q(t, \cdot)\right\|_{2}}
$$

and define the overall control law

$$
u^{\star}(t, x)=u_{\circ}(t, x)+v_{b}(t, x) .
$$

Remark 5 Let us notice that the introduction of the discontinuous term (8) might be somewhat problematic in principle. Indeed, the derivation of the Lyapunov function as in (4) requires the existence of a sufficiently smooth solution to the parabolic equation, e.g. a strong solution, and the classical regularity theory does not apply in this case, where the notion of approximate (and thus weak) solutions has to be introduced. On the other hand, as discussed later on in Section 4, the problem can be shown to be well posed by direct inspection. In this perspective, let us preliminarily claim and assume that a unique solution of the system driven by the control input (9) exists, and that the differentiation operations in (4) are allowed.

Theorem 2 Let us consider the perturbed equation (3), where the disturbance input $b(t, x)$ satisfies Assumption 3. Then the sliding-mode control input (9) guarantees robust asymptotic convergence of the solution $q(t, x)$ to the stationary solution $\{q=0\}$, i.e.

$$
\lim _{t \rightarrow+\infty}\left\|h(t, \cdot)-h^{\star}(t, \cdot)\right\|_{2}=0 .
$$

PROOF. Following the steps of the proof of Proposition 1 , under the action of the control input $u_{\circ}$, the derivative $\dot{V}$ reduces to

$$
\begin{aligned}
\dot{V}(q(t, \cdot)) & \leq-\left(\lambda_{\min } C_{\mathrm{P}}+\eta\right) \int_{\Omega}|q|^{2} d x \\
& +\int_{\Omega} b \cdot q d x-M_{b} \int_{\Omega} \frac{P_{\mathcal{W}} q \cdot q}{\left\|P_{\mathcal{W}} q(t, \cdot)\right\|_{2}} d x
\end{aligned}
$$

Let us focus on the last two terms. By construction one has

$$
\int_{\Omega} b q d x=\int_{\Omega} b \cdot P_{\mathcal{W}} q d x+\underbrace{\int_{\Omega} b \cdot P_{\mathcal{W}} q d x}_{=0}
$$

and

$\int_{\Omega} \frac{P_{\mathcal{W}} q \cdot q}{\left\|P_{\mathcal{W}} q(t, \cdot)\right\|_{2}} d x=\int_{\Omega} \frac{P_{\mathcal{W}} q \cdot P_{\mathcal{W}} q}{\left\|P_{\mathcal{W}} q(t, \cdot)\right\|_{2}} d x=\left\|P_{\mathcal{W}} q(t, \cdot)\right\|_{2}$.

Now, using the Hölder's inequality, one gets

$$
\int_{\Omega} b \cdot P_{\mathcal{W}} q d x \leq\|b(t, \cdot)\|_{2} \cdot\left\|P_{\mathcal{W}} q(t, \cdot)\right\|_{2}
$$

this showing that, thanks to Assumption 3, the following bound is enforced

$$
\begin{aligned}
& \int_{\Omega} b \cdot q d x-M_{b} \int_{\Omega} \frac{P_{\mathcal{W}} q \cdot q}{\left\|P_{\mathcal{W}} q(t, \cdot)\right\|_{2}} d x \\
\leq & -\left(M_{b}-\|b(t, \cdot)\|_{2}\right)\left\|P_{\mathcal{W}} q(t, \cdot)\right\|_{2} \leq 0 .
\end{aligned}
$$

Summarizing, we find again the estimate

$$
\dot{V}(q(t, \cdot)) \leq-\left(\lambda_{\min } C_{\mathrm{P}}+\eta\right) \int_{\Omega}|q|^{2} d x,
$$

which proves that the discontinuous control $v_{b}(t, x)$ is capable to cancel out the effect of disturbances on the system dynamics, guaranteeing robust asymptotic stability.

\section{Approximate solutions VS Regularized sliding-modes}

As already mentioned, the well-posedness of the differential problem in the presence of a discontinuous input term is a delicate issue. Singular terms in partial differential equations typically require ad-hoc treatments, depending on the nature of singularity. For instance, natural tools for handling terms where singularity in the space variable appear are the classical Hardy-Sobolev inequality [11] and the so-called Carleman estimates [12]. Conversely solution-dependent singularities, such as the 
one arising in the infinite dimensional sliding-mode control (7), can be handled by introducing the concept of approximate solutions, which is inherited from [25] and reported hereafter.

Definition 1 A continuously differentiable function $h^{\delta}(t, x)$ is an approximate $\delta$-solution to problem (3)-(9) if it is a strong solution of the corresponding problem where the discontinuous term $v_{b}(t, x)$ is replaced by an approximation $v_{b}^{\delta}(t, x)$ with

$$
\left\|v_{b}(\cdot, \cdot)-v_{b}^{\delta}(\cdot, \cdot)\right\|_{2,2} \leq \delta, \quad\left\|h^{\delta}(\cdot, \cdot)\right\|_{2,2} \geq \delta
$$

Furthermore, Problem 1 is said to admit a generalized solution $\breve{h}$ if there exists a family of approximate solutions $\left\{h^{\delta}(t, x)\right\}_{\delta \in \mathbb{R}^{+}}$such that

$$
\lim _{\delta \rightarrow 0}\left\|h^{\delta}(t, \cdot)-\breve{h}(t, \cdot)\right\|_{2}=0
$$

uniformly in $t \in[0, \tau)$.

Rather than considering approximate solutions, whose implementation might be though and computationally demanding, we propose an alternative approach based on regularization of the sliding-mode by means of cutoff functions that remove the singularities.

For any fixed $\epsilon>0$, we define the continuously differentiable function $\varrho_{\epsilon}:[0, \infty) \rightarrow[0,1]$ as

$$
\varrho_{\epsilon}(s)= \begin{cases}1 & s \geq \epsilon \\ \frac{-16(s-\epsilon)^{2}}{2 \epsilon^{2}}+1 & s \in(3 \epsilon / 4, \epsilon) \\ \frac{16(s-\epsilon / 2)^{2}}{2 \epsilon^{2}} & s \in(\epsilon / 2,3 \epsilon / 4] \\ 0 & s \leq \epsilon / 2\end{cases}
$$

The proposed control strategy is then given by the following family of state-feedback laws, obtained as a parameter-dependent regularization of an infinitedimensional sliding mode:

$$
u_{\epsilon}(t, x)=u_{\circ}(t, x)+\tilde{v}_{b, \epsilon}(t, x)
$$

where $\tilde{v}_{b, \epsilon}(t, x)$ is obtained from (8) by introducing a

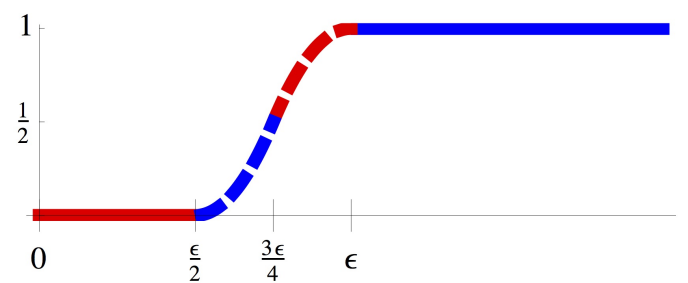

Fig. 1. Cut-off function cut-off multiplier and a compensation term:

$$
\begin{aligned}
\tilde{v}_{b, \epsilon}(t, x)= & -M_{b} \varrho_{\epsilon}\left(\left\|P_{\mathcal{W}} q(t, \cdot)\right\|_{2}\right) \frac{P_{\mathcal{W}} q(t, x)}{\left\|P_{\mathcal{W}} q(t, \cdot)\right\|_{2}} \\
& -M_{b} P_{\mathcal{W} \perp} q(t, x)
\end{aligned}
$$

The following result is then straightforward to prove.

Proposition 6 For any fixed $\epsilon>0$, the nonsingular control input (12) guarantees robust attractivity and forward invariance of the set

$$
\mathbb{V}_{\epsilon, \mathcal{W}}:=\left\{q(\cdot) \in W_{0}^{1,2}(\Omega): \begin{array}{l}
\left\|P_{\mathcal{W}} q(t, \cdot)\right\|_{2} \leq \epsilon \\
\left\|P_{\mathcal{W}^{\perp}} q(t, \cdot)\right\|_{2} \leq \sqrt{\epsilon}
\end{array}\right\}
$$

i.e. the origin is a robustly practically stable equilibrium for the strong solution of the error system driven by such control input.

PROOF. Mimicking the steps of the proof of Theorem 2 with (8) replaced by (12), one gets the inequality

$$
\begin{aligned}
\dot{V}(q(t, \cdot)) & \leq-\left(\lambda_{\min } C_{\mathrm{P}}+\eta\right) \int_{\Omega}|q|^{2} d x \\
& +\int_{\Omega} b \cdot q d x-M_{b} \int_{\Omega} P_{\mathcal{W} \perp} q \cdot q d x \\
& -M_{b} \varrho_{\epsilon}\left(\left\|P_{\mathcal{W}} q(t, \cdot)\right\|_{2}\right) \int_{\Omega} \frac{P_{\mathcal{W}} q \cdot q}{\left\|P_{\mathcal{W}} q(t, \cdot)\right\|_{2}} d x
\end{aligned}
$$

The right-hand side is clearly negative definite as long as $\left\|P_{\mathcal{W}} q(t, \cdot)\right\|_{2} \geq \epsilon$, this yielding attractivity and invariance of the unbounded set $\mathbb{V}_{\epsilon, \mathcal{W}}^{1}:=\left\{q(\cdot) \in W_{0}^{1,2}(\Omega)\right.$ : $\left.\left\|P_{\mathcal{W}} q(\cdot)\right\|_{2} \leq \epsilon\right\}$. On the other hand, whenever the solution lies in the set $\mathbb{V}_{\epsilon, \mathcal{W}}^{2}:=\mathbb{V}_{\epsilon, \mathcal{W}}^{1} \backslash \mathbb{V}_{\epsilon, \mathcal{W}}$, one has

$$
\begin{aligned}
& \int_{\Omega} b \cdot q d x-M_{b} \int_{\Omega} P_{\mathcal{W} \perp} q \cdot q d x \\
= & \int_{\Omega} b \cdot P_{\mathcal{W}} q d x-M_{b} \int_{\Omega}\left(P_{\mathcal{W} \perp} q\right)^{2} d x \\
\leq & M_{b}\left\|P_{\mathcal{W}} q(t, \cdot)\right\|_{2}-M_{b}\left\|P_{\mathcal{W} \perp} q(t, \cdot)\right\|_{2}^{2} \\
\leq & M_{b}\left(\epsilon-(\sqrt{\epsilon})^{2}\right)=0,
\end{aligned}
$$

which yields again

$$
\dot{V}(q(t, \cdot)) \leq-\left(\lambda_{\min } C_{\mathrm{P}}+\eta\right) \int_{\Omega}|q|^{2} d x .
$$

In conclusion, the practical stability within the region $\mathbb{V}_{\epsilon, \mathcal{W}}$ has been established.

Remark 7 It is worth to emphasize that for any $\epsilon>0$ the set $\mathbb{V}_{\epsilon, \mathcal{W}}$ is compact, i.e. closed and bounded, and 


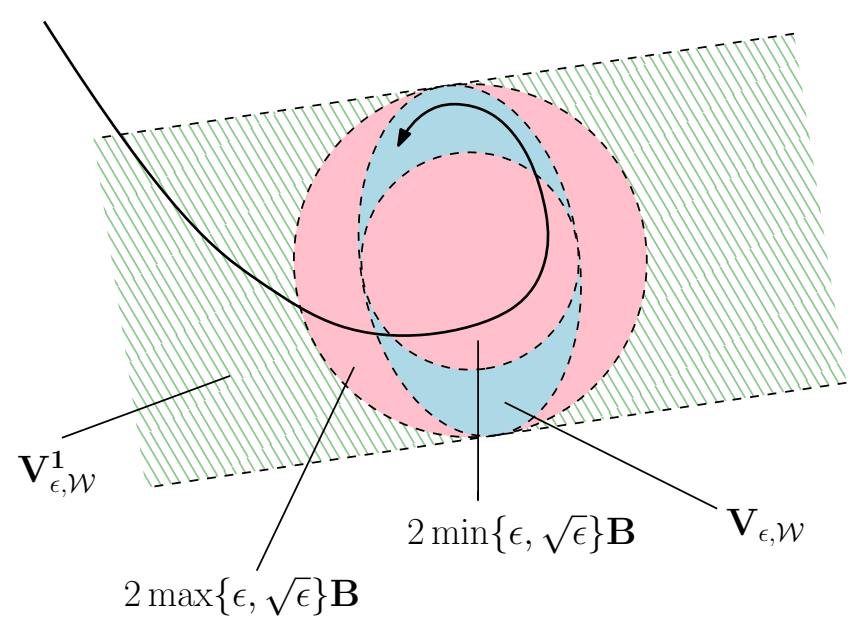

Fig. 2. Graphical illustration in the $\left(\mathcal{W}, \mathcal{W}^{\perp}\right)$-hyperplane of the region of attraction provided by the regularized control input (11).

moreover the following inclusions hold:

$$
2 \min \{\epsilon, \sqrt{\epsilon}\} \mathbb{B} \subset \mathbb{V}_{\epsilon, \mathcal{W}} \subset 2 \max \{\epsilon, \sqrt{\epsilon}\} \mathbb{B}
$$

A graphical representation is proposed in Figure 2.

\section{$5 \quad$ Numerical tests}

Let us set $\Omega=[0,2 \pi]$ and consider the unidimensional Fisher-KPP equation with non-homogeneous diffusion in divergence form

$$
\partial_{t} h=\partial_{x}\left(a(t, x) \partial_{x} h\right)+0.3 h(1-h)+u(t, x)+b(t, x)
$$

with

$$
a(t, x)=15 \arctan \left(1+(\sin t)^{2} x^{2}\right)
$$

which verifies the uniform ellipticity condition (2) with $\lambda_{\min }=15 \arctan (1)$ and $\lambda_{\max }=15 \arctan \left(1+4 \pi^{2}\right)$. The initial/boundary conditions as imposed as follows:

$$
\begin{aligned}
& h(0, x)=0.2 x(x-2 \pi) \\
& h(t, 0)=-\sin (2 t), \quad h(t, 2 \pi)=\sin (2 \pi-2 t) .
\end{aligned}
$$

The reference to be tracked is assigned in the form of the traveling wave $h^{\star}(t, x)=\sin (x-2 t)$, which constitutes an interesting solution profile [23]. Moreover, the external perturbation $b(t, x)=5 \cos 2 t \cos x$ has been considered, with bound $\|b(t, \cdot)\|_{2} \leq M_{b}=5 \sqrt{\pi}$. We notice that $b(t, x)$ belongs for $t \geq 0$ to the closed subspace $\mathcal{W}$ of zero-mean functions, i.e.

$$
\mathcal{W}=\left\{\varpi(\cdot) \in \mathcal{L}^{2}(0,2 \pi): \int_{0}^{2 \pi} \varpi(x) d x=0\right\}
$$

Setting $\widehat{\varpi}=\left(\int_{0}^{2 \pi} \varpi(x) d x\right) /(2 \pi)$, the linear operators $P_{\mathcal{W}}$ and $P_{\mathcal{W}}^{\perp}$ are defined as follows:

$$
P_{\mathcal{W} \varpi}(x)=\varpi(x)-\widehat{\varpi}, \quad P_{\mathcal{W}}^{\perp}=\widehat{\varpi}
$$

Four different scenarios have been simulated, namely:

a) No control input, without disturbance

b) Tracking controller (7), without disturbance

c) Tracking controller (7), with disturbance

d) Sliding-mode controller (11), with disturbance

As shown in Figure 3, when no control action is taken the behavior of the solution $h(t, x)$ does not comply with the desired reference $h^{\star}(t, x)$. Conversely, when the feed-forward control law is implemented, a quiet accurate reference tracking is achieved, as clearly visible in Figure 4. If the disturbance term $b(t, x)$ is plugged in the equation, the nominal input (7) is no longer able to guarantee the desired reference tracking task, see Figure 5. However, the sliding-mode controller, as expected by the theoretical guarantees developed in Theorem 2 and Proposition 6, is capable to ensure robust practical stability. The control performances can be appreciated in Figure 6, where the regularized version (11) has been implemented with a tolerance parameter $\epsilon=0.2$. The practical stability is further illustrated in Figure 7, where the norm of the tracking error is plotted for different values of the parameter $\epsilon$. Let us point out that the norm of the initial error is $\|q(0, x)\|_{2} \approx 4$.

\section{Conclusions}

This paper addresses the problem of robust distributed tracking control for quasilinear reaction-diffusion equations in higher-dimensional spatial domains. The main contributions of the work are the introduction of novel stabilizing control laws based on quantitative integral inequalities, the design of sliding-mode inputs through the projection onto a infinite-dimensional manifold and the synthesis of a novel regularization technique for achieving practical stabilization.

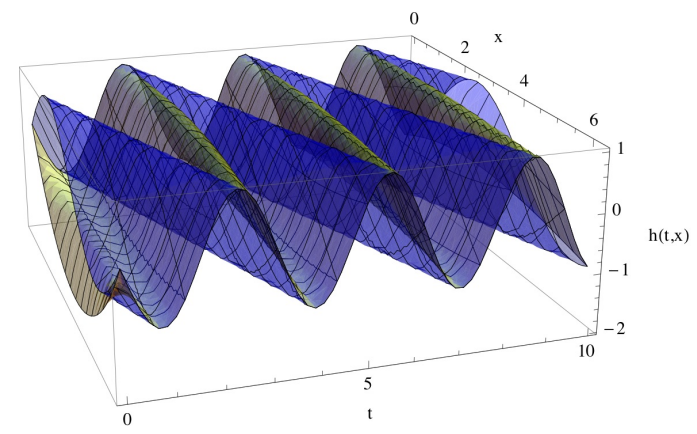

Fig. 3. Example a), uncontrolled case: evolution of $h(t, x)$ (yellow) and $h^{\star}(t, x)$ (blue) 


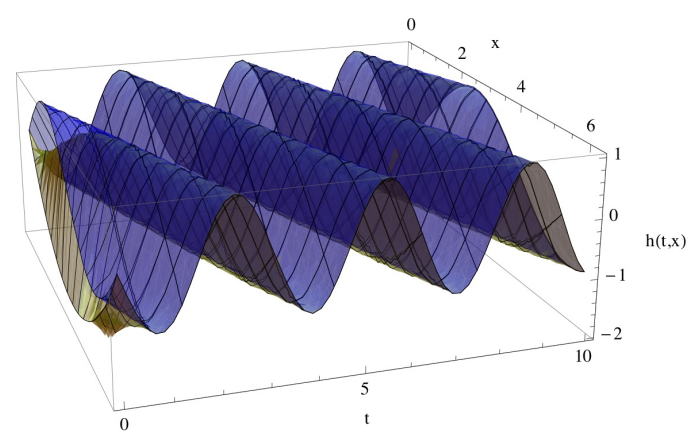

Fig. 4. Example b), feed-forward tracking controller: evolution of $h(t, x)$ (yellow) and $h^{\star}(t, x)$ (blue)

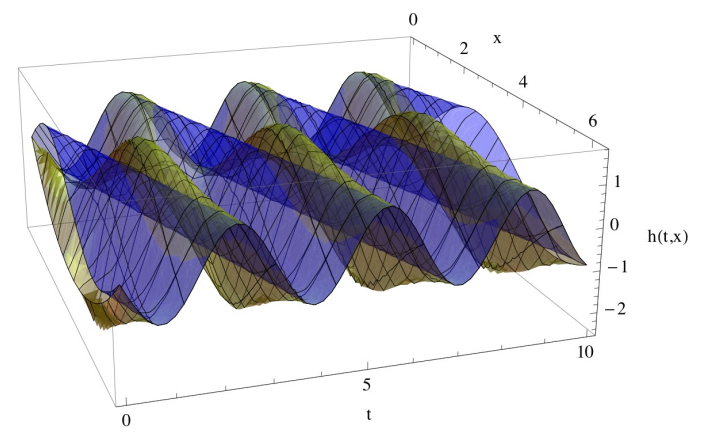

Fig. 5. Example c), feed-forward tracking controller with external disturbance: evolution of $h(t, x)$ (yellow) and $h^{\star}(t, x)$ (blue)

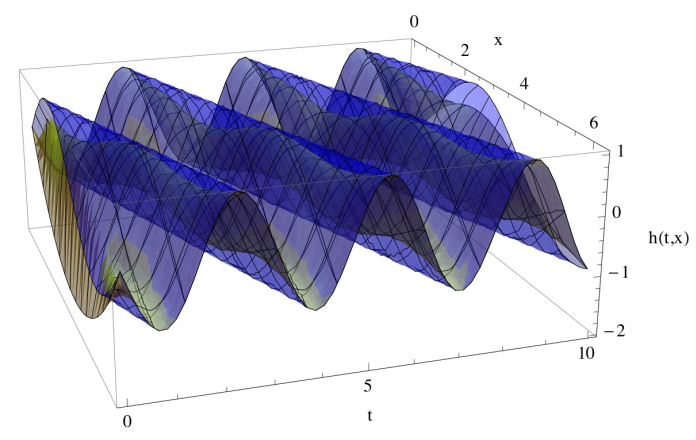

Fig. 6. Example d), robust control $u_{\epsilon}(t, x)$ : evolution of $h(x, t)$ (yellow) and $h^{\star}(t, x)$ (blue)

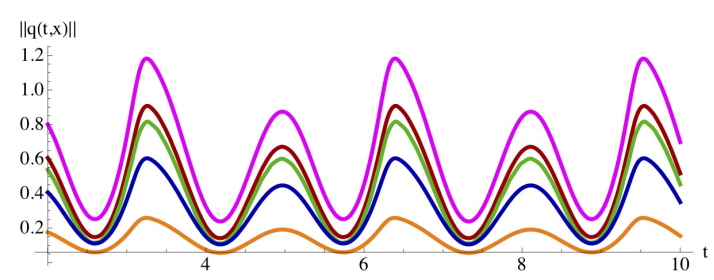

Fig. 7. Example d), practical stability: $\mathcal{L}^{2}$-norm of tracking error for $\epsilon=0.5$ (magenta), $\epsilon=0.3$ (red), $\epsilon=0.2$ (green), $\epsilon=0.1$ (blue) and $\epsilon=0.02$ (orange)

\section{References}

[1] R.A. Adams and J.J.F. Fournier. Sobolev spaces, volume 140. Academic press, 2003.

[2] T. Ahmed-Ali, F. Giri, M. Krstic, F. LamnabhiLagarrigue, and L. Burlion. Adaptive observer for a class of parabolic pdes. IEEE Transactions on Automatic Control, 61(10):3083-3090, 2016.

[3] W. Barthel, J. Christian, and F. Tröltzsch. Optimal boundary control of a system of reaction diffusion equations. Zeitschrift für Angewandte Math. und Mech., 90(12):363-382, 2010.

[4] D. Bresch-Pietri and M. Krstic. Output-feedback adaptive control of a wave pde with boundary antidamping. Automatica, 50(5):1407-1415, 2014.

[5] H. Brezis. Functional analysis, Sobolev spaces and partial differential equations. Springer, Berlin, 2010.

[6] C.I. Bymes, D.S. Laukó, I.G.and Gilliam, and V.I . Shubov. Output regulation for linear distributed parameter systems. IEEE Transactions on Automatic Control, 45(12):2236-2252, 2000.

[7] A. Cristofaro. Robust tracking control for a class of perturbed and uncertain reaction-diffusion equations. IFAC Proceedings Volumes, 47(3):1137511380, 2014.

[8] J. Deutscher. Output regulation for linear distributed-parameter systems using finitedimensional dual observers. Automatica, 47(11):2468-2473, 2011.

[9] J. Deutscher. A backstepping approach to the output regulation of boundary controlled parabolic pdes. Automatica, 57:56-64, 2015.

[10] L. C. Evans. Partial differential equations. AMS, Providence, 1998.

[11] S. Filippas, V.G. Maz'ya, and A. Tertikas. Sharp Hardy-Sobolev inequalities. Comptes Rendus Mathematique, 339(7):483-486, 2004.

[12] G. Fragnelli and D. Mugnai. Carleman estimates and observability inequalities for parabolic equations with interior degeneracy. Advances in Nonlinear Analysis, 2(4):339-378, 2013.

[13] E. Fridman and Y. Orlov. An LMI approach to $H_{\infty}$ boundary control of semilinear parabolic and hyperbolic systems. Automatica, 45:2060-2066, 2008.

[14] P. Grindrod. The theory and applications of reaction-diffusion equations: Patterns and waves. Clarendon, Oxford, 1996.

[15] A. Hasan, O. M. Aamo, and B. Foss. Boundary control for a class of pseudo-parabolic differential equations. Systems \& Control Letters, 62(1):63-69, 2013.

[16] D. Jones, M. Plank, and B. Sleeman. Differential equations and mathematical biology. CRC Press, 2010.

[17] M. Kishida and R. D. Braatz. Structured spatial control of the reaction-diffusion equation with parametric uncertainties. In Proc. of 2010 IEEE Int. Symposium on Computer-Aided Control System Design, pages 1097-1102, 2010. 
[18] M. Krstic and A. Smyshlyaev. Adaptive boundary control for unstable parabolic PDEs-Part I: Lyapunov design. IEEE Transactions on Automatic Control, 53(7):1575, 2008.

[19] M. Krstic and A. Smyshlyaev. Adaptive control of PDEs. Ann. Rev. Control, 32:149-160, 2008.

[20] S. Marx, E. Cerpa, C. Prieur, and V. Andrieu. Global stabilization of a Korteweg-De Vries equation with saturating distributed control. SIAM J. on Control and Optimization, 55(3):1452-1480, 2017.

[21] V. Maz'ya. Sobolev spaces. Springer, 2013.

[22] T. Meurer and A. Kugi. Tracking control for boundary controlled parabolic PDEs with varying parameters: Combining backstepping and differential flatness. Automatica, 45(5):1182-1194, 2009.

[23] G. Nadin and L. Rossi. Propagation phenomena for time heterogeneous KPP reaction-diffusion equations. Journal de Mathématiques Pures et Appliquées, 98(6):633-653, 2012.

[24] Y. Orlov. Application of lyapunov method in distributed systems. Automation and Remote Control, 44(4):426-431, 1983.

[25] Y. Orlov. Discontinuous systems Lyapunov analysis and robust synthesis under uncertainty conditions. Springer, Berlin, 2009.

[26] Y. Orlov, A. Pisano, and E. Usai. Continuous statefeedback tracking of an uncertain heat diffusion process. Systems \& Control Letters, 59(12):754-759, 2010.

[27] Y. Orlov and V. I. Utkin. Unit sliding mode control in infinite dimensional systems. Applied Mathematics and Computer Science, 8(1):7-20, 1998.

[28] L. Paunonen and S. Pohjolainen. Internal model theory for distributed parameter systems. SIAM Journal on Control and Optimization, 48(7):47534775, 2010.

[29] A. Pisano, Y. Orlov, and E. Usai. Tracking control of the uncertain heat and wave equation via powerfractional and sliding-mode techniques. SIAM J. Control Optim., 49(2):363-382, 2011.

[30] G. Schneider and H. Uecker. Nonlinear PDEs: A Dynamical Systems Approach, volume 182. American Mathematical Soc., 2017.

[31] H. Sira-Ramirez. Distributed sliding-mode control in systems described by quasilinear partial differential equations. Systems $\&$ Control Letters, 13:177$181,1989$.

[32] V.I. Utkin. Sliding modes in control and optimization. Springer, Berlin, 1992.

\section{Appendix A - Review of some classical inequali- ties in functional spaces}

For the readers' convenience a collection of classical inequalities that have been used in the paper are reported.

Poincaré's inequality. Let $v(x) \in W_{0}^{1, p}(\Omega), p \geq 1$. There exists a positive constant $C_{\mathrm{P}}>0$ (depending on $p, n$ and $\Omega$ ) such that

$$
\|v\|_{p}^{p} \leq C_{\mathrm{P}}\|\nabla v\|_{p}^{p}
$$

Jensen's inequality. Let $h(x) \in L^{1}(\Omega)$ be non-negative with $|\Omega|<\infty$, and consider a continuous, convex and non-negative function $\varphi: \mathbb{R}^{+} \rightarrow \mathbb{R}^{+}$. Then the following estimate holds:

$$
\varphi\left(\frac{1}{|\Omega|} \int_{\Omega} h(x) d x\right) \leq \frac{1}{|\Omega|} \int_{\Omega} \varphi(h(x)) d x .
$$

Gagliardo-Nirenberg-Sobolev inequality. Let $v(x) \in$ $W_{0}^{1, p}(\Omega)$ and $1 \leq p<n$. There exists a positive constant $C_{\mathrm{S}}>0$ such that

$$
\|v\|_{p^{*}}^{p^{*}} \leq C_{\mathrm{S}}\|\nabla v\|_{p}^{p}
$$

where $p^{*}>p$, referred to as the Sobolev conjugate of $p$, is given by the relationship $1 / p^{*}=1 / p-1 / n$.

For a detailed discussion about these inequalities and their connections with more general properties of Sobolev spaces, one may refer to the textbooks $[1,21]$.

\section{Appendix B - An interpolation inequality}

Let $m, k$ be real numbers with $0<m<k$. Then one has

$$
|z|^{m} \leq \frac{1+|z|^{k}}{\gamma(m, k)} \quad \forall z \in \mathbb{R}
$$

where

$$
\gamma(m, k):=\left(\frac{1}{\left(\frac{m}{k}\right)^{\frac{m}{k-m}}-\left(\frac{m}{k}\right)^{\frac{k}{k-m}}}\right)^{1-\frac{m}{k}}>0
$$

PROOF. Without loss of generality we can focus on the case $z \geq 0$. Let us define the function $f(z)=1+z^{k}-\gamma z^{m}$, where $\gamma>0$ is a constant to be specified. One has

$$
f(0)=1>0, \quad \lim _{z \rightarrow+\infty} f(z)=+\infty,
$$

this showing that the minimum of $f(z)$ must be attained in some point $z_{o} \in(0,+\infty)$. On the other hand, imposing

$$
f^{\prime}(q)=q^{m-1}\left(k q^{k-m}-\gamma m\right)=0
$$

a unique stationary point $z_{o}$ is found in $(0,+\infty)$, namely

$$
z_{o}=\left(\gamma \frac{m}{k}\right)^{\frac{1}{k-m}}
$$

The expression (13) for $\gamma$ is then obtained evaluating $f(z)$ in $z=z_{o}$ and imposing $f\left(z_{o}\right)=0$. 